\title{
Left Atrium Dimension
}

National Cancer Institute

\section{Source}

National Cancer Institute. Left Atrium Dimension. NCI Thesaurus. Code C99970.

The diameter across the left atrium measured at the end of systole. (ACC) 